\section{Hydroformylation of Styrene Derivatives Catalyzed by Rhodium Single-Atoms Supported on $\mathrm{CeO}_{2}$}

Polymer-Supported

Synthesis

\section{Key words}

rhodium catalysis

hydroformylation

single-atom catalyst

styrenes<smiles></smiles>

(50 mg)

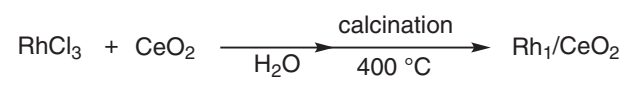

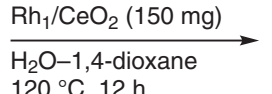

(3 MPa)<smiles></smiles>

linear (I)<smiles></smiles>

branched (b)
Results:<smiles>O=CCCc1ccccc1</smiles>

conversion: $55 \%$ yield $\mathrm{l} / \mathrm{b}=3.0$<smiles>CC(CC=O)c1ccccc1</smiles>

conversion: $47 \%$ yield selectivity for aldehyde: $80 \%$ yield $\mathrm{l} / \mathrm{b}=12$<smiles>C=Cc1ccccc1</smiles>

(50 mg) selectivity for aldehyde: $75 \%$ yield<smiles>O=CCCc1ccc(Cl)cc1</smiles>

conversion: $94 \%$ yield selectivity for aldehyde: $61 \%$ yield $\mathrm{l} / \mathrm{b}=2.7$<smiles>O=CCC(O)c1ccccc1</smiles><smiles>C=C(Br)c1ccccc1</smiles>

conversion: $99 \%$ yield selectivity for aldehyde: $77 \%$ yield $\mathrm{l} / \mathrm{b}=4.0$

$$
\mathrm{l} / \mathrm{b}=3.0
$$<smiles>Cc1ccc(CCC=O)cc1</smiles>

conversion: $49 \%$ yield selectivity for aldehyde: $72 \%$ yield
Significance: A rhodium single-atom catalyst on $\mathrm{CeO}_{2}\left(\mathrm{Rh}_{1} / \mathrm{CeO}_{2}\right)$ was prepared by mixing $\mathrm{RhCl}_{3}$ and $\mathrm{CeO}_{2}$ in deionized water, followed by calcination at $400{ }^{\circ} \mathrm{C}$ (eq. 1). $\mathrm{Rh}_{1} / \mathrm{CeO}_{2}$ catalyzed the hydroformylation of styrene derivatives with hydrogen generated in situ from water and carbon monoxide to give the corresponding linear aldehydes in $\leq 99 \%$ conversion (eq. 2).
Comment: $\mathrm{Rh}_{1} / \mathrm{CeO}_{2}$ was characterized by means of ICP-OES, STEM, HAADF-STEM, FT-IR, EDS analyses. In the hydroformylation of styrene, Rh nanoparticles supported on $\mathrm{CeO}_{2}\left(\mathrm{NP}-\mathrm{Rh} / \mathrm{CeO}_{2}\right)$ gave 3-phenylpropan-1-ol rather than 3-phenylpropanal, with $99 \%$ selectivity (eq. 3 ). 